\title{
ENCUENTRO DE NOCIONES PROPIETARIAS. PLEITOS POR TIERRA EN GUACARHUE, CHILE, 1820-1850*
}

\author{
MEETING OF PROPRIETARY NOTIONS. LAND TRIALS IN GUACARHUE, \\ CHILE, 1820-1850
}

\author{
Víctor Brangier ${ }^{* *}$ y Mauricio Lorca***
}

\begin{abstract}
El estudio aborda la convergencia tensa de nociones propietarias que pequeños y medianos campesinos sostuvieron en litigios por el acceso a la tierra. Se escogió como escenario de observación el valle de Guacarhue, espacio agrícola de la zona central de Chile que desde 1820 experimentó la tensión por la formalización de derechos a la tierra. A partir de un estudio de caso, se constató que los actores, argumentando en atención a intereses de acceso respecto de este bien, posibilitaron el encuentro de nociones propietarias disímiles. Por un lado, entró en escena el sustento que la propiedad tuvo en experiencias de residencia, poblamiento y trabajo, como legado de la cultura jurisdiccional del periodo colonial. Por otro, figuró la emergente validez exclusiva de la escrituración pública. El análisis corrobora la práctica litigiosa como instancia de redefinición y promoción de nociones propietarias. La originalidad de la propuesta radica en la posibilidad de trascender los usos institucionales que los actores hicieron respecto de las políticas de tierra de los nuevos Estados en América Latina, como se ha sostenido hasta el momento. Posibilita, en cambio, comprender estas experiencias litigantes como uno de los ejes fundantes de esas políticas.
\end{abstract}

Palabras claves: Tierra, propiedad, siglo XIX, Chile, justicia.

The study addresses the tense convergence of proprietary notions that small and medium farmers held in litigation to access land. The Guacarhue Valley, an agricultural space in the central area of Chile, which, since 1820, underwent tension due to the formalization of land rights, was chosen as the observation scenario. A case study, found that the actors, arguing in response to access interests about this real state, made it possible to find dissimilar proprietary notions. On the one hand, it arose on the basis that the property had in residences experiences, settlement and work, as a legacy of the jurisdictional culture of the colonial period. On the other hand, there was the emerging exclusive validity of the public deed. The analysis corroborates the litigious practice as an instance of redefinition and promotion of proprietary notions. This proposal's originality lies in the possibility of transcending the institutional uses that the actors made concerning land policies of the new states in Latin America, as has been maintained so far. It allows, however, to understand these litigating experiences as one of the foundational axes of those policies.

Key words: Land, property, XIXth century, Chile, justice.

\section{Introducción}

La monarquía ibérica administró sus dominios ultramarinos mediante la delegación de potestad en autoridades territoriales. Desde virreyes a alcaldes y corregidores tenían entre sus misiones el gobierno de las poblaciones mediante la mantención de la paz. Para lograr este propósito en un régimen jurídico plural, las autoridades debían conservar los derechos desiguales de cada quien. De ese modo, la administración de justicia resultó ser una de las principales facultades delegadas por el rey (Barriera 2013). Gestionando un ejercicio más político que técnico-jurídico, los agentes territoriales de la monarquía debían garantizar la tranquilidad del vecindario allanando conflictos por medio de la escucha de las partes y el reconocimiento de sus posiciones sociales y jurídicas. Finalmente debían recurrir a su arbitrio para dirimir a base de la conveniencia y al bien común. La justicia tenía como fin la paz de los desiguales, marco ético que orientaba el gobierno local (Agüero 2008; Del Arenal y Speckman 2009). Este modelo específico de administración de espacios y poblaciones, en que gobierno y justicia devinieron gimnasia indisociable, ha sido reconocido como política jurisdiccional (Garriga 2004).

Para garantizar el bien común, la monarquía ibérica recurrió preferentemente a la distribución de tierras. En el marco de esta política jurisdiccional en Hispanoamérica, las autoridades locales cedieron tierras bajo el régimen de mercedes para retribuir proporcionalmente los méritos de la conquista.

\footnotetext{
* Resultado del Proyecto de Investigación Interno 2020 de la Universidad Bernardo O’Higgins (UBO/VVCMEI2003).

** Centro de Estudios Históricos, Universidad Bernardo O’Higgins. Santiago. Correo electrónico: vmbrangi@ gmail.com

*** Instituto de Investigaciones en Ciencias Sociales y Educación, Universidad de Atacama. Copiapó, Chile.

Correo electrónico: mauricio.lorca@uda.cl
} 
Durante el régimen colonial, la cesión estuvo condicionada a tres requisitos que debía cumplir el beneficiario para sincronizar el beneficio individual con el bien común: la residencia, el poblamiento y el trabajo (Mariluz 1978; Dougnac 1998). Así se conjuraba el riesgo de la acumulación improductiva, asegurando el acceso social a la tierra y la tributación a la Corona.

$\mathrm{El}$ advenimiento de las independencias trajo aparejada una nueva política de tierras. La inserción progresiva de la producción agrícola a los circuitos mercantiles internacionales incrementó el valor de este bien. Desde entonces se retomó la iniciativa de delimitación agraria que el régimen borbón impulsó en la centuria anterior para conocer el territorio a administrar y propulsar la agricultura (Garavaglia y Marchena 2005). Con estos mismos fines y con las urgencias de restaurar las arcas fiscales vía tributación propietaria, las autoridades de los Estados independientes aspiraron a la formalización de derechos de los bienes raíces. Las ideas jurídicas propietarias, provenientes del Código Civil napoleónico, orientaron en un primer momento los esfuerzos que apuntaban a la generación de propietarios con derechos reales sobre la tierra. La posesión de un instrumento público de dominio y su posterior inscripción en un registro nacional, tornaba a su titular en dueño soberano, condicionado a los preceptos mínimos de no transgredir las leyes del Estado (Guzmán 2015; Jara 2016). Así, se anulaban las antiguas exigencias de residencia, poblamiento y trabajo que restringían y a la vez orientaban el dominio.

Considerando esta colisión de concepciones propietarias, este artículo problematiza las instancias de encuentro, hibridación y desplazamiento gradual entre ambas. Se ha escogido un escenario de observación, caracterizado por la tensión creciente entre los actores ligados a la tierra, debido al alza en el valor de este bien, la necesidad de precisar los derechos a su uso y goce y la asimilación social de aquellas nociones que legitimaban la posesión y el dominio. El escenario fue el valle agrícola de Guacarhue, situado en el valle central de Chile y distante 123 kilómetros al sur de Santiago. El sector fue parte de las primeras encomiendas dada a los militares de la conquista en el siglo XVI y conformó una gran estancia de lenta subdivisión a lo largo de las centurias coloniales (León 1991). Guacarhue integró la provincia de Colchagua, área de vocación triguera y ganadera desde el siglo XVIII, atravesada por grandes haciendas circundadas por economías campesinas dependientes (Cáceres 2007; Robles 2003). Atendiendo este último aspecto, el objetivo del artículo apunta a examinar el modo en que pequeños y medianos campesinos vehiculizaron sus intereses de acceso a la tierra mediante la apelación a estas nociones propietarias disímiles. Resulta de particular interés el despliegue y reformulación de estos presupuestos en situación judicial.

El periodo de análisis se extiende entre 1820 y 1850 . El primer límite temporal marcó el fin de las batallas de independencia que tuvieron a Guacarhue como uno de los teatros predilectos de las confrontaciones (León 1991). Desde entonces y lentamente, se retomaron las actividades agrícolas y comerciales en la zona y, junto con ello, se incrementaron los pleitos por el acceso y exclusión de derechos sobre la tierra. La década de 1850, por otro lado, marcó el inicio del boom triguero en la provincia de Colchagua, con la inclusión de la economía triguera a los mercados internacionales y el alza exponencial del valor de la tierra (Cariola y Sunkel 1982). Además, la década culmina con la instalación del primer registro conservador de bienes raíces en el sector, en la villa de Rengo, 17 kilómetros al este de Guacarhue. Desde entonces advino una nueva noción propietaria, reflejada en la teoría y práctica de la posesión inscrita, según estableció el Código Civil chileno promulgado en 1855 (Barahona 2016). Los actores vinculados a la tierra no solo debían resguardar su dominio mediante la celebración de escrituras públicas por medio de notarios y jueces locales, según se practicaba hasta el momento. A partir esa fecha, resultaba necesario inscribir esos documentos en la oficina del registro conservador.

\section{Las nociones propietarias frente a la justicia de jueces}

Para identificar el empleo que pequeños y medianos campesinos realizaron de distintas concepciones propietarias en situación judicial, se recurrió al Archivo Nacional Histórico de Chile [ANHCh], Fondo Judicial de San Fernando, sección Causas Civiles [FJSC]. Entre los cientos de expedientes judiciales disponibles, se seleccionaron aquellos juicios que involucrara a residentes del valle de Guacarhue durante el periodo seleccionado ${ }^{1}$. El material se descubrió en este Fondo debido a que el juzgado de letras de la ciudad de San Fernando, el 
único de esa naturaleza en la provincia de Colchagua, archivó los litigios tramitados por los jueces legos del territorio, incluidos los del juzgado de alcalde de Guacarhue (Brangier 2019).

Del conjunto de expedientes, se escogieron disputas por acceso y exclusión de derechos sobre la tierra, sostenidas por actores de medianos y pequeños recursos agrícolas. Para definir la pertenencia de los litigantes a la categoría de pequeños y medianos campesinos, se recurrió a información que proporcionaron los expedientes y, además, se confrontó su participación en ejercicios de transferencias de tierras (compra, ventas, herencias y donaciones). Esta documentación se encuentra en protocolos notariales del Archivo Nacional de la Administración de Chile [ARNAD], Fondo de Notarios de Rengo [FNR]. La triangulación de estos criterios permitió levantar una muestra de 14 expedientes judiciales.

La documentación fue analizada a partir del enfoque de la construcción histórica de la propiedad, según ha enfatizado la ius historiografía agrícola. Como la historiadora Rosa Congost ha expuesto en varios trabajos (Congost 2019; Congost y Santos 2010), la aproximación a las nociones y praxis propietarias de un tiempo y espacio determinado, debe atender más a las correlaciones de fuerzas políticas y sociales y a los acuerdos y conflictos derivados de allí, que a concepciones formalmente grabadas en el derecho positivo (aunque considerando a este último como uno de los factores). En este sentido, la indagación historiográfica no solo debe ser sensible a los desplazamientos entre formas propietarias sucesivas, sino también a las convergencias e hibridaciones (Elickson 1993; Barcos 2013; Congost 2019). En esta investigación, este presupuesto contribuyó al escrutinio del encuentro de nociones propietarias sobre la tierra, en los argumentos de pequeños y medianos campesinos en situación judicial. Considerando que en un mismo tiempo y lugar se reunió y tensó "un haz de derechos" de carácter plural (Poczynok 2018) el examen documental permitió rescatar la recurrencia litigante a nociones propietarias disímiles.

A partir del enfoque anterior, en estas páginas se emplea el concepto de "nociones propietarias" para referirse a los fundamentos y paradigmas acerca de los derechos al uso y goce legítimos sobre la tierra que los litigantes esgrimieron en situación judicial. Se presupone que la condición de posibilidad del despliegue de estos paradigmas fue el carácter de la justicia de jueces más que de leyes (Lorente
2007; Brangier y Barriera 2015). De este modo, el estudio considera un segundo presupuesto derivado de la ius historiografía del derecho y desde donde se rescatan los ejes medulares de la política jurisdiccional, según han sido sintetizados más arriba. Estos aportes permiten subrayar que en Chile, como en otras latitudes de la región, tras las coyunturas independentistas continuó la vinculación entre política, administración y justicia. En la mayoría de los territorios de las repúblicas nacientes, el ejercicio judicial estaba a cargo de jueces legos y vecinos (Yangilevich 2012). En Chile se replicó el modelo y, desde el reglamento de administración de justicia de 1824 en adelante, la justicia de menor y mínima cuantía quedó a cargo de alcaldes, subdelegados e inspectores, ante la escasa presencia de jueces letrados (Brangier, Matheu y Ovalle 2019). Esta justicia vecinal explica el despliegue y validación de nociones propietarias diversas en los juzgados, por medio de la que pequeños y medianos campesinos intentaban vehiculizar sus intereses relativos a los derechos sobre la tierra.

La indagación de la muestra documental, basada en estos enfoques y conceptos, permitió seleccionar un expediente judicial representativo con el que se construyó el estudio de caso. El juicio develó, de manera más nítida que el resto de la documentación, el encuentro de nociones propietarias dispares. El pleito duró cinco años desde 1829 , pero fue antecedido por un conflicto intrafamiliar de 26 años. La selección estuvo determinada porque la variedad de actores en el litigio sostuvieron argumentos y decisiones judiciales en torno a los dos argumentos de legitimidad de derechos a la tierra que convergían en estas décadas: por un lado, las experiencias de residencia, poblamiento y trabajo y, por otra, la posesión de escrituras de titulación. Las partes, sus representantes, los alcaldes territoriales y los jueces letrados partícipes manifestaron comprender la vigencia de estas nociones propietarias a lo largo de tres décadas de conflicto. Unos y otros construyeron sus razonamientos y decisiones reconociendo la validez y vigencia de estas experiencias que sustentaban la argumentación litigante.

\section{Treinta y tres años de experiencias y nociones propietarias plurales}

En 1801 Carlos Trincado, propietario de 128 cuadras continuas de tierra en la localidad de Guacarhue, testó en favor de sus diez hijos. Al 
fallecer ese año, sus herederos convocaron jueces partidores para realizar un inventario y distribución de los bienes. A todos ellos se les dejó en distintas porciones equivalentes, colindantes y sin deslindes ni cercamiento. Dos años después, uno de los hijos, Marcelo Trincado, traspasó a su cuñado Manuel Ahumada, cuatro y media cuadras de tierra a cambio de 30 pesos. Esta transferencia se transformó en el inicio de un extenso conflicto al interior de la familia que motivó este pleito judicial. A lo largo del juicio, Ahumada argumentó que el traspaso fue una venta, mientras que Marcelo Trincado sostuvo que se trató de un empeño y, por tanto, que su cuñado debía devolver la tierra una vez que recibiera de vuelta los 30 pesos.

En 1821 Dionisia Trincado, esposa de Ahumada, falleció intestada. Este solicitó al Dr. Diego José de Argomedo la elaboración del inventario de sus bienes $^{2}$. Manuel y Dionisia tuvieron nueve hijos y todos ellos recibieron una porción de las tierras, según el inventario. El documento incluyó dentro de estos bienes las cuatro cuadras y media que Marcelo Trincado le había traspasado 18 años antes a Manuel Ahumada. Además, fueron reconocidas como bienes del matrimonio otras cuadras que Ahumada adquirió en distintos momentos de dos de los hermanos Trincado. Según el expediente judicial, desde 1803 Manuel Ahumada estuvo 26 años poseyendo, invirtiendo y realizando mejoras en estos territorios.

En julio de 1829 Marcelo Trincado demandó a Ahumada ante Gregorio Ramírez, alcalde de la villa de San Fernando, solicitando la restitución de las cuatro cuadras y media de tierras e intentando frenar una posesión que, a su juicio, resultaba ilegítima. Con la demanda se inició el juicio referido. El alcalde Ramírez ordenó a José Valenzuela y Velazco, alcalde de segunda elección de San Fernando, que llamara a conciliación ${ }^{3}$. Inmediatamente Trincado nombró como representante a su yerno Alejo Piña, oficial del regimiento miliciano de caballería. El 31 de julio de ese año, el edil Valenzuela y Velazco convocó a las partes a su despacho. El informe de conciliación señaló que ambos mantuvieron sus respectivas versiones. De todos modos, Manuel Ahumada reconoció que su cuñado ya le había devuelto los 30 pesos y, por tanto, se comprometía a restituir las tierras. Sin embargo, añadió, como él y su familia vivían en las cuatro cuadras y media en disputa y además las tenía sembradas y les había hecho mejoras en los últimos 26 años, ofrecía el equivalente a ese terreno en otro sector ${ }^{4}$. Alejo Piña se negó y sostuvo la demanda por las tierras originales. Ante la imposibilidad de avenencia, el alcalde Valenzuela y Velazco dictaminó que, como Ahumada recibió los 30 pesos, debía abandonar las tierras y que un tasador midiera las mejoras e inversiones para que se le paguen en compensación (ANHCh, FJSFC 1829: 10).

Tras el decreto de restitución, Alejo Piña desalojó con violencia a Ahumada y su familia, debiendo trasladarse a unos ranchos que levantó de improviso. Así quedó en evidencia en los descargos que hizo Manuel Ahumada, al mes siguiente, frente a Gregorio Ramírez, alcalde de primera elección de San Fernando. En este punto del expediente se deduce que Ahumada era un agricultor de cortos recursos. En efecto, desde entonces comenzó a litigar como pobre y aseguró que el despojo le había perjudicado "sus cortas siembras" el contexto general del pleito, también se infiere una política constante de aumento de capital mediante adquisición de pequeñas porciones de tierras a los hermanos Trincado. Esto último quedó patente en la declaración de Alejo Piña ante el alcalde de San Fernando en septiembre de 1829. Tras insistir que las cuatro cuadras y media correspondían legítimamente a su suegro por herencia, agregó que, a propósito de la partición de bienes de 1801 "los jueces partidores de la herencia no definieron ni amojonaron bien los lindes, por indiferencia o porque no se solía hacer entonces" (ANHCh, FJSFC 1829: 20). Manuel Ahumada, aprovechándose de esa distribución imprecisa, habría adquirido tierras de sus cuñados herederos, generalmente mediante acuerdos verbales. Según añadía Alejo Piña, Ahumada se posesionaba y sembraba el terreno tras cada adquisición para volver irreversible el dominio ${ }^{6}$.

El expediente indicó que el juicio se mantuvo sin acciones por dos años. En ese intervalo se revirtió el despojo y Ahumada recuperó las tierras, volviendo a habitar y a sembrar. No quedó claro el modo ni las acciones de este retorno, pero en abril de 1831 el conflicto volvió a foja cero y Manuel Ahumada seguía ocupando las tierras. Ese mes, nueve de los hermanos Trincado, herederos originales del terreno, solicitaron a José Marful, alcalde de Guacarhue, una nueva partición de los bienes de Carlos Trincado, con tal de precisar la distribución de 1801. El 21 de abril todos ellos comparecieron en el despacho del edil. También estaba Manuel Ahumada. En conjunto acordaron reabrir el testamento de Carlos 
Trincado redactado 30 años antes y realizar una nueva mensura de las tierras heredadas. Con este fin, los hermanos herederos nombraron a Marful como "juez compromisario, árbitro, arbitrador, y amigable componedor", mientras que Manuel Ahumada designó a Ignacio de los Reyes como juez partidor (ANHCh, FJSFC 1829: 27).

Los jueces partidores realizaron la mensura y emitieron el informe un mes después, en mayo de 1831. Allí ratificaron la dimensión de 128 cuadras de la herencia en su conjunto. El reporte culminó con la repartición del terreno entre los diez herederos legítimos, incluyendo a Manuel Ahumada como representante y albacea de su difunta esposa Dionisia Trincado. Según los partidores, a todos ellos "se les puso en posesion del terreno que les correspondia, dandoseles tanto bueno, como tanto malo (...) y sumaron 9 cuadras y media por heredero. Se fijaron los linderos que marcaron las hijuelas" (ANHCh, FJSFC 1829: 28). El informe contuvo las rúbricas de conformidad de los hermanos Trincado y de Manuel Ahumada.

El pleito continuó, no obstante el nuevo acuerdo de distribución. Manuel Ahumada no consideró válido el último informe de partición porque desconocía los acuerdos de adquisiciones que había pactado con distintos hermanos Trincado y las inversiones agrícolas realizadas en esas tierras. Pese a que el documento contuvo la firma de Ahumada, en los hechos, este continuó habitando y trabajando las porciones de terreno que gozaba previo a la nueva partición. Desde ese momento, Alejo Piña, como representante y yerno de la contraparte, decidió cambiar de estrategia judicial. Hasta ese momento la demanda sobre las tierras que ocupaba Ahumada se basaba en argumentos de adquisición engañosa y en el cuestionamiento a la partición original de los bienes heredados. Desde este momento, la demanda se centró exclusivamente en la existencia y validez de los documentos que certificaban las adquisiciones. Por ello, tres meses después, en agosto de 1831, Piña compró cinco cuadras de tierra a uno de los hermanos Trincado, José Antonio. La compra incluía propiedades que Ahumada poseía desde hace 26 años, por eventual compra que hizo en 1805 al actual vendedor. En esta oportunidad, Piña se aseguró que la transacción quedara registrada en instrumento público redactado por el alcalde Marful ${ }^{7}$.

En septiembre de 1831 las partes litigaron ante Alejandro Sepúlveda, alcalde de la cercana villa de Rengo. Alejo Piña presentó a tres hermanos Trincado como testigos de las adquisiciones ilegítimas de Ahumada. Uno de ellos, José María, refirió que su cuñado también se posesionó paulatinamente de los terrenos que correspondían a su hermano Estanislao y aseguró que escuchó de este "no haberle vendido, ni harrendado jamás el terreno que Ahumada le tiene tomado" (ANHCh, FJSFC 1829: 21). De ese modo, Alejo Piña subrayaba que en el punto en que se encontraba la causa, su cuñado no contaba con documentos que acreditaran sus adquisiciones y además el informe de los partidores decretaba la ilegitimidad de su posesión. Manuel Ahumada intentó mantener el litigio en los argumentos de adquisición por acuerdo y de posesión legítima. Reiteró al alcalde que, mediante acuerdos verbales compró y arrendó tierras de distintos hermanos Trincado a lo largo de estas décadas con el fin de cercarlas y trabajarlas.

Dos meses después, en noviembre de 1831, la causa se elevó al juzgado de letras de San Fernando. El juez Pedro María de la Arriagada puso en estado de probanza. Manuel Ahumada presentó un interrogatorio que debían contestar cuatro testigos. Las preguntas apuntaban a corroborar que, durante las últimas tres décadas, Ahumada ocupó y mejoró las cuatro cuadras y media que adquirió de manos de Marcelo Trincado, como además otras tierras de sus cuñados. El juez de letras derivó el interrogatorio al alcalde de Rengo quien pasó al domicilio de los testigos. Las declaraciones confirmaron la versión de Manuel Ahumada. Uno de los testigos era Marcos Trincado, de 60 años, reconocido como uno de los hermanos herederos. El otro fue José Canales, de 70 años, padrino de José Antonio Trincado. De ese modo, quedaba en evidencia que Ahumada solo mantenía conflicto con una parte de la familia Trincado. Hasta ese momento, lograba movilizar a la otra parte familiar a declarar en su favor. Sin embargo, la estrategia argumentativa de Ahumada seguía girando en torno a la legitimidad de la posesión, apoyada en la trayectoria de habitación y producción de los terrenos adquiridos. Por el contrario, la ofensiva de Alejo Piña profundizó la nueva estrategia que pretendía la exhibición de documentos públicos que certificaran las adquisiciones. Con este fin, ofreció al juzgado de letras un interrogatorio cuyas preguntas informaban acerca de las compras irregulares, secretas y de mala fe que hizo en su momento la contraparte. Los tres testigos (uno de ellos era Carlos Trincado, otro de los hermanos herederos) ratificaron que a lo largo 
de 30 años, Ahumada expandió de facto la herencia original de 1801 que correspondió a su esposa. Alejo Piña concluyó su probanza indicándole al juez letrado que, específicamente respecto de las cinco cuadras que le vendió José Antonio Trincado, él gozaba de la escritura de compra firmada por el alcalde Marful, mientras que Ahumada solo contaba con un relato de una compra sin certificación realizada en 1805.

El juicio se detuvo por siete meses. El 26 de junio de 1832 Manuel Ahumada otorgó poder de representación en José Parga, quien inmediatamente escribió al juez refiriendo que las pruebas de Alejo Piña resultaban inválidas. Parga demostró comprender la estrategia del demandante e intentó reforzar la posición de Ahumada arguyendo que, no obstante haber puntualizado lo contrario, las adquisiciones que su representado hizo a los hermanos Trincado si tenían escrituras públicas que las certificaran. El problema era recuperarlas, debido al tiempo que había transcurrido y a la precariedad de los archivos de los jueces territoriales que actuaban en nombre de escribanos ausentes. A los oídos de los contemporáneos debía resultar un razonamiento verosímil, atendiendo la fragilidad o inexistencia de archivos judiciales de los jueces legos del territorio (Brangier 2019; Brangier y Barriera 2015). En relación con las cinco cuadras de tierras de José Antonio Trincado, Parga indicó que Ahumada las compró en 1805 mediante instrumento público redactado por José Ramírez, entonces alcalde de Guacarhue. El documento habría quedado archivado en el despacho del edil. Las guerras de independencia se desplegaron en esta zona y el archivo se destruyó junto con la escritura.

El tres de septiembre de 1832 Alejo Piña respondió a los descargos de la contraparte. Parecía cómodo en el nuevo terreno probatorio en el que se desenvolvía el juicio y apuntó a invalidar el argumento contrario de la escritura de compra extraviada. Entonces solicitó a Alejandro Sepúlveda, alcalde de Rengo, interrogara al antiguo alcalde José Ramírez, para probar la veracidad de la tesis de José Parga. Sepúlveda interrogó a Ramírez quien señaló que no hubo escritura pública que certificara la compra y que él no supo de aquella transacción (ANHCh, FJSFC 1829: 62). Inmediatamente, Piña escribió al juez de letras argumentando ilegitimidad en la posesión de Ahumada ante la falta de certificaciones de adquisición.

El nuevo juez de letras de San Fernando, Pedro José Jaramillo, retomó la causa siete meses después.
Tras revisión de los antecedentes, el magistrado sentenció que debía respetarse el decreto que pronunció cuatro años antes el alcalde de segunda elección de San Fernando. En este sentido, Manuel Ahumada debía devolver las cuatro cuadras y medias que adquirió de Marcelo Trincado considerando que este le había devuelto los 30 pesos de su valor. Además, Trincado debía compensar a su cuñado por las mejoras que había realizado en las tierras durante todos estos años (ANHCh, FJSFC 1829: 75). La sentencia no hizo mención al resto de las adquisiciones que Ahumada habría hecho informalmente a los hermanos Trincado y que se disputaban en la demanda. Finalmente, Manuel Ahumada recurrió a la Corte de Apelaciones de Santiago. La causa estuvo allí un año y medio sin movimiento hasta que el 13 de mayo de 1834, los ministros ratificaron la sentencia anterior (ANHCh, FJSFC 1829: 96).

\section{Discusión: redefiniciones litigantes de la propiedad de la tierra}

El juicio descrito dialoga con las corrientes de estudios relativos a la política jurisdiccional que primó en Hispanoamérica desde el periodo fundacional. El estudio de caso aproxima a las transformaciones de este modelo durante las primeras décadas de vida independiente. De acuerdo con el nuevo espíritu republicano y liberal, el bien común dejaba de orientar implícitamente el ejercicio de gobernantes-jueces y ahora cristalizaba en la ley como expresión escrita y pública del contrato social (Bravo 2006). Como en Europa continental, tras la ruptura con el Antiguo Régimen, la política en estas latitudes ya no requería conservar un orden social y jurídico establecido originalmente, sino que debía impulsar la sociedad hacia la felicidad y el progreso, como versión renovada del bien común (Prodi 2008). Los gobiernos que aspiraban hacia este nuevo fin emprendieron una política de tierras orientada hacia la restauración económica y, de paso, pretendieron solventar las alicaídas arcas fiscales mediante tributos a la renta agrícola (Garavaglia y Marchena 2005). El plan resultaba coherente con la adopción de presupuestos basados en la noción de propiedad plena, pues, según el credo liberal, el ciudadano se tornaría más productivo al contar con certezas respecto de los derechos reales sobre sus bienes (Congost y Santos 2010). Estas convicciones derivarían de la titulación pública de los derechos 
propietarios, deviniendo en adelanto agrícola y bienestar de la sociedad. Resultaba necesario desplazar cualquier condicionante de esas certidumbres, como los antiguos requisitos de residencia, poblamiento y trabajo que determinó la iniciativa regia de cesión condicionada de tierras.

Los resultados del estudio enriquecen esta línea de discusión. Se ha insistido al respecto que en la región, las autoridades de los nuevos estados, junto con las elites terratenientes locales y los juristas, empujaron el proceso de formalización de derechos sobre la tierra (Brooke 2004; Banzato, Barcos y D'Agostino 2013). En efecto, en zonas de nueva colonización, la formalización benefició a los especuladores en desmedro de antiguos ocupantes y colonos productores (Gelman y Santilli 2013). En las áreas de antigua colonización, los ocupantes quedaron atrapados como dependientes de la gran propiedad que extendía sus límites formales (Mörner 1974). El pleito descrito más arriba, en cambio, demuestra que las distintas concepciones propietarias convergieron y fueron reinterpretadas y disputadas con intensidad en escenarios judiciales. En la medida que los actores siguieron intereses concretos de acceso a derechos sobre la tierra y desplegaron estrategias litigantes en situación judicial, leyeron y aplicaron estos presupuestos propietarios disímiles, contribuyendo a sus redefiniciones y desplazamientos.

Respecto de contextos latinoamericanos similares, se ha señalado que los sujetos ligados a la producción agroganadera emprendieron un uso social de la justicia y de las nociones jurídicas propietarias emergentes para conservar el acceso a la tierra (Escobar y Butler 2013; Moraes, 2015). En este sentido, los hallazgos descritos devienen originales porque constatan que los actores sociales, mediante argumentación litigante, impulsaron las tensiones y reconfiguraciones de estas nociones, tanto antiguas como emergentes. Los sujetos en situación judicial contornearon el perfil de estas ideas y no solo se limitaron a ensayar un uso judicial de argumentos técnico-jurídicos preestablecidos ${ }^{8}$.

Para confirmar el punto anterior, conviene resaltar algunos elementos del juicio. La pugna derivó de una mensura y partición de bienes de la herencia de Carlos Trincado, quien, tras fallecer en 1801, dejó 128 cuadras de tierra a sus 10 hijos. Según se comentó, los jueces partidores distribuyeron entre los hermanos Trincado sin precisar los lindes. Una práctica similar demostró el juez partidor Diego José de Argomedo 20 años más tarde, cuando Manuel Ahumada le solicitó realizar un inventario de los bienes de su esposa difunta para distribuir entre sus hijos. El juez incluyó en el documento tierras que Ahumada había adquirido de sus cuñados a partir de acuerdos verbales y posesiones de facto, prescindiendo de escrituras de titulación. Estas prácticas evidencian la relevancia que los jueces y residentes del medio asignaban a la posesión y al trabajo de las tierras como elemento legitimador del dominio. Manuel Ahumada residía con su familia en estos terrenos que había adquirido y que había sembrado. A ojos de los jueces partidores, tanto en 1801 como en 1821, esos elementos otorgaban mayores derechos a la tierra que la escritura pública que verificara la adquisición.

Sin embargo, la demanda por restitución de tierras que emprendió Marcelo Trincado contra su cuñado Manuel Ahumada en 1829, develó la asimilación social a una nueva concepción propietaria. La estrategia judicial de Trincado y de Alejo Piña, su yerno y representante, tendió a validar las escrituras públicas de adquisición como evidencia propietaria exclusiva. Desde esa nueva ribera argumentativa intentaron cuestionar la posesión de la contraparte y deslegitimar las experiencias de residencia, poblamiento y trabajo como fuentes del dominio. La inclusión de este presupuesto tensionó los presupuestos propietarios de los litigantes, representantes y jueces involucrados en el juicio. En efecto, la determinación de José Valenzuela y Velazco, alcalde de San Fernando, tras el intento fallido de conciliación en 1829, demostró un reconocimiento a la inversión agrícola que Ahumada realizó sobre los terrenos durante 26 años. El decreto de desalojo que emitió el edil, al mismo tiempo que consideró la necesidad de escrituras públicas como certificación de dominio, incluyó el imperativo de compensación económica al demandado tras tasación de la inversión. Este razonamiento fue replicado por Jaramillo, juez de letras de San Fernando cuatro años más tarde, dictaminando que debía respetarse la resolución del alcalde Valenzuela y Velazco. Además omitió cualquier referencia a las demandas sobre derechos ilegítimos que Ahumada mantendría sobre otras tierras adquiridas a los hermanos Trincado?.

Los actores del juicio calibraron la tensión entre las nociones propietarias en disputa. Los jueces convocados por las partes en 1831 para generar una nueva partición, simplemente decidieron borrar toda experiencia de posesión y dominio y redistribuir 
equitativamente. La determinación implicaba un reconocimiento a la imposibilidad de resolver el intríngulis considerando los fundamentos del dominio que alegaban las partes. A juicio del alcalde de Guacarhue, José Marful y del juez partidor Ignacio de los Reyes, resultaba de mayor conveniencia renovar el pacto de la familia Trincado sobre sus derechos a la tierra. De todos modos, a lo largo del juicio hubo voces sensibles al predominio de la titulación por sobre la posesión y el trabajo. Así lo demostró José Parga, quien desde 1832 actuó como representante de Manuel Ahumada frente al juez de letras de San Fernando. Parga prefirió descartar la tesis sostenida por Ahumada en tres años de pleito y mantuvo el debate exclusivamente en la arena de titulación. Entonces recurrió al supuesto que su representado sí adquirió las tierras de su cuñado José Antonio Trincado mediante escritura pública en 1805, pero esta se extravió de los archivos del alcalde de Guacarhue, José Ramírez Molina. Ese mismo año, el alcalde de Rengo, Alejandro Sepúlveda, también reconoció el predominio propietario de la titulación por sobre las experiencias de posesión de Ahumada. Por ello respondió con prontitud a la demanda de Alejo Piña para que interrogara al alcalde Ramírez Molina respecto de la veracidad de los supuestos de Parga.

El estudio de caso devela la importancia telúrica de los litigios sobre derechos a la tierra para la redefinición y desplazamiento de nociones propietarias. De todos modos, como evidencia el juicio examinado, resultó también imprescindible la actitud que presentaron las autoridades territoriales con potestad judicial. Tanto alcaldes, como jueces partidores y jueces de letras, escucharon a las partes y, mediante dictámenes, legitimaron o hibridaron sus argumentos. Sin esta comunicación de los agentes de los juzgados, con los presupuestos y estrategias de las partes, las nociones propietarias de estos últimos no hubiesen tenido cabida. Este aspecto se torna inteligible si se considera que los agentes de los juzgados, incluyendo a los representantes de las partes, pertenecían al mismo medio social y cultural de los litigantes. Así quedó diseñado desde los primeros intentos de formulación del poder judicial en Chile en la década de 1820 (Brangier, Matheu y Ovalle 2019). Los operadores judiciales bebían, por tanto, de las mismas fuentes de información y participaban de los circuitos de ideas e intereses de los justiciables, manteniendo así, uno de los aspectos medulares de la política jurisdiccional del periodo colonial. De esta justicia de jueces más que de leyes (Lorente 2007), derivaba la sensibilidad de los operadores de los juzgados respecto de las nociones propietarias que portaban los litigantes.

\section{Conclusión}

La propuesta problematizó la convergencia y tensión de nociones propietarias disímiles, en un contexto agrícola en que estas concepciones se consideraban excluyentes. Por un lado, se puso atención a la vigencia de los fundamentos que el dominio sobre la tierra vinculaba a la residencia, al poblamiento y al trabajo. Por otro, se resaltó la emergencia de la titulación como exigencia exclusiva de la propiedad plena. De ese modo, se pretendió examinar el empleo que pequeños y medianos campesinos realizaron de estas nociones en situación judicial para catalizar sus intereses de acceso a la tierra. Se abordó un estudio de caso, rescatado de una muestra de pleitos judiciales producidos en el valle de Guacarhue entre 1820 y 1850, en los que se disputaba el acceso a y exclusión de derechos sobre la tierra. El expediente judicial develó un extenso conflicto intrafamiliar por las formas de adquisición de la tierra y sus derechos a uso y goce. Los litigantes, representantes y jueces corroboraron la asimilación creativa de ambas nociones de propiedad, generando encuentros e hibridaciones. El valle de Guacarhue, como escenario de la confrontación, manifestó disposición de los jueces a atender y validar los presupuestos y estrategias de las partes. En una coyuntura crítica de formalización de derechos, esta condición operativa, reconocida como justicia de jueces, resultó axial para posibilitar el encuentro entre ambas nociones.

La originalidad de esta propuesta descansa en la posibilidad de aproximarse a la reformulación social de nociones vinculadas a la propiedad de la tierra. La literatura que abordó el contexto de formalización de derechos, debatió entre la exclusión social a este bien en que derivó el proceso y la instrumentalización institucional que los campesinos realizaron para garantizar derechos. Sin embargo, en estas páginas se constató que en situación judicial de carácter jurisdiccional, los litigantes reescribieron estas nociones. En este sentido, esta propuesta requiere abordajes adicionales que examinen el modo en que estas redefiniciones en situación judicial, conectaron con las políticas públicas sobre la tierra. Resulta de alto interés complementar estos 
hallazgos con el análisis comparado y conectado de las nociones propietarias que fundamentaron las iniciativas estatales, nacionales y locales respecto del acceso a la tierra. Sobre todo, considerando el proceso dinámico de ensayo y error que caracterizó estas políticas durante el siglo XIX en la región. Un abordaje de este tipo requiere la identificación de correas de transmisión entre los escenarios judiciales-locales de tensión de ideas propietarias y las esferas jurídicas y políticas de decisión.

\section{Referencias Citadas}

Agüero, A.

2008 Castigar y perdonar cuando conviene a la república. La justicia penal de Córdoba del Tucumán, siglos XVII y XVIII. Centro de Estudios Políticos y Constitucionales, Madrid.

Albornoz, M.

2014 "Casos de corte y privilegios de pobreza: lenguajes jurídicos coloniales y republicanos para el rescate de derechos especiales en el momento de litigar por injurias. Chile, 1700-1874". Signos Históricos 32: 48-85.

Albornoz, M.

2019 "Peritos en medir, dibujar y definir las tierras. Notas para el estudio y catastro de los agrimensores en Chile, siglos XVI-XIX”. Revista Historia y Justicia 13: 1-30.

Banzato, G.; Barcos, M.; D’Agostino, V.

2013 "Problemas, métodos y abordajes teóricos en torno al mercado de tierras. La campaña bonaerense entre los siglos XVIII y XIX”. En Tierras Rurales. Políticas, transacciones y mercados en Argentina, 1780-1914, dirigido por G. Banzato, pp. 19-63. Rosario, Prohistoria.

Barahona, C.

2016 "La teoría de la posesión inscrita en Chile en el siglo XIX (1857-1910)". Tesis para optar al grado de Magíster. Programa de Magíster en Derecho, Universidad de Chile, Santiago.

Barriera, D.

2013 Abrir puertas a la tierra. Microanálisis de la construcción de un espacio político. Santa Fe, 1573-1640. Ministerio de Innovación y Cultura de la Provincia de Santa Fe - Museo Histórico Provincial "Brigadier Estanislao López", Santa $\mathrm{Fe}$.

Brangier, V.

2019 Saber hacer y decir en justicia. Culturas jurídicojudiciales en la zona centro sur de Chile. 1824-1875. Prohistoria, Rosario.

Brangier, V.; Matheu, A.; Ovalle, A.

2019 "Puentes sobre la tierra. Estrategias de brokers en una red de venta de tierra. Pichidegua, Chile, 1820-1845". Diálogo Andino 60: 9-19.

Brangier, V. y Barriera, D.

2015 'Lenguajes comunes en 'justicia de jueces'. Tratamientos historiográficos y fondos judiciales en Chile y Argentina". Humanidades 32: 227-258.

Bravo, B.

2006 "Protección jurídica de los gobernados frente a los gobernantes en el Nuevo Mundo (1492-1992). De los recursos judiciales a las garantías constitucionales". En El juez entre el derecho y la ley. Estado de Derecho y Derecho de Estado en el mundo hispánico, siglos XVI a XXI, compilado por B. Bravo, pp. 201-253. Santiago: Lexis Nexis.
Brooke, L.

2004 Trials of Nation Making. Liberalism, Race and Ethnicity in the Andes, 1810-1910. Cambridge University Pres, Cambridge.

Cáceres, J.

2007 Poder Rural y Estructura Social. Colchagua, 17601860. La Construcción del Estado y la Ciudadanía desde la Región. Universidad Católica de Valparaíso, Valparaíso.

Cariola, C. y Sunkel, O.

1982 La Historia Económica de Chile, 1830 y 1930: dos Ensayos y una Bibliografía. Instituto de Cooperación Interamericana, Madrid.

Congost, R.

2019 "Las leyes sobre la propiedad de la tierra y el análisis histórico de los derechos de propiedad. Reflexiones a partir de algunos bicentenarios". Claves. Revista de Historia 1, 1: 121-137.

Congost, R. y Santos, R.

2010 "From formal institutions to the social contexts of property". In Context of Property in Europe. The Social Embeddedness of Property Rights in Land in Historical Perspective, edited by R. Congost \& R. Santos pp. 15-38. Turnhout: Brepols.

Del Arenal, J y Speckman, E. (Coords.)

2009 El mundo del derecho. Aproximaciones a la cultura jurídica novohispana y mexicana (siglos XIX y XX). UNAM, Instituto de Investigaciones Históricas, Escuela Libre de Derecho, México.

Dougnac, A.

1998 Manual de Historia del Derecho Indiano. Segunda edición. UNAM, México.

Elickson, R.

1993 "Property in land". Yale Law School. Faculty Scholarship Series 411: 219-304.

Escobar, A.; Butler, M.

2013 "Introduction. Transitions and Closures in Nineteenth-and Twentieth- Century Mexican Agrarian History". In México in Transition: New Perspectives on Mexican Agrarian History, Nineteenth and Twentieth Centuries, edited by A. Escobar and M. Butler, pp. 33-76. México, CIESAS.

Garavaglia, J. y Marchena, J.

2005 América Latina. De los orígenes a la Independencia. II. La sociedad colonial ibérica en el siglo XVIII. Crítica, Barcelona.

Garriga, C.

2004 Orden jurídico y poder político en el Antiguo Régimen. Istor IV, 16: 13-44.

Gelman, J.; Santilli, D.

2013 Movilidad social y desigualdad en el Buenos Aires del siglo XIX: el acceso a la propiedad de la tierra entre el rosismo y el orden liberal. Hispanic American Historical Review 93, 4: 659-684. 
Guzmán, A.

2015 "La tradición como modo de adquirir el dominio en el derecho romano, en el común y en el iusnaturalismo y su destino en los derechos patrios de la América española”. Revista Chilena de Derecho 42, 1: 329 - 344.

Jara, M.

2016 "El artículo 925 del Código Civil. La verdadera querella de amparo". Revista de Derecho 29, 2: 109-130.

León, R.

1991 Quinta de Tilcoco. Alborada de un pueblo. Conyre, Santiago.

Lorente, M. (Ed.)

2007 De Justicia de Jueces a justicia de leyes: hacia la España de 1870. Consejo General del Poder Judicial, Madrid.

Mariluz, J.

1978 El régimen de la tierra en el derecho indiano. Perrot, Buenos Aires.

Mellafe, M.

1988 "Prólogo". En Historia de la reforma agraria en Chile, editado por J. Garrido pp. 18-30. Santiago: Universitaria.

Moraes, J.

2015 "A terra em disputa: câmara municipal versus juízes comissários". Unisinos 19, 2:229-241.

Mörner, M.

1974 "La hacienda Hispanoamericana en la historia: un esquema de reciente investigación y debate". Desarrollo económico 13, 52: 741-773.

Poczynok, C.

2018 "La propiedad de la tierra como un haz de derechos. Una contribución desde la campaña bonaerense en un momento transicional (1800-1829)". Mundo Agrario 19, 41: 1-20.

Prodi, $\mathrm{P}$.

2008 Una historia de la justicia. De la pluralidad de fueros al dualismo moderno entre conciencia y derecho. Katz, Madrid.

Robles, C

2003 "Expansión y transformación de la agricultura en una economía exportadora: la transición al capitalismo en Chile (1850-1930)". Historia agraria: revista de agricultura e historia rural 29: 45-80.

Undurraga, V.

2015 "Presentación del dossier Formas de conciliación y mecanismos informales de resolución de conflictos en Chile, siglos XVII a XIX". Revista de Historia Social y de las Mentalidades 19, 2: 1-2.

Yangilevich, $\mathrm{M}$.

2012 Estado y criminalidad en la frontera sur de Buenos Aires (1850-1880). Prohistoria, Rosario.

\section{Fuentes}

Fondo de Contaduría Mayor. Segunda serie, volumen 3742. Archivo Nacional Histórico.

Fondo de Notarios de Rengo. Volúmenes 18 y 24. Archivo Nacional de la Administración.

Fondo Judicial de San Fernando. Caja 79. Expediente 16. Archivo Nacional Histórico de Chile.

Fondo Judicial de San Fernando. Caja 42. Expediente 15. Archivo Nacional Histórico de Chile.

\section{Notas}

1 La etapa de identificación de expedientes judiciales contó con la participación de tres estudiantes de la carrera de Pedagogía en Historia y Geografía en Enseñanza Media de la Universidad Bernardo O’Higgins: Josefa López, Catalina Ramírez y Catalina Espinoza. Las tres estudiantes se incluyeron en el equipo de Personal Técnico del Proyecto de Investigación Interno 2020 de la Universidad Bernardo O'Higgins (UBO/VVCMEI2003).

2 La familia Argomedo representaba uno de los principales grupos terratenientes y comerciantes de Colchagua desde el siglo XVIII. Diego José de Argomedo integró este clan y fue uno de los electores por San Fernando para la elección de diputados de 1813 (Cáceres 2007).

3 Verónica Undurraga (2015) se aproximó a los juicios de conciliación en Chile tradicional, como vía resolutiva de conflictos interpersonales.

4 Un intento de acuerdo similar registró otro caso de la muestra, en que vecinos colindantes del sector de Tilcoco fueron llamados a conciliación por el alcalde de Guacarhue (ANHCH, FJSF 1848)

5 De todos modos conviene precisar que la figura jurídica y judicial de declaratoria de pobreza reflejaba menos una condición socioeconómica de los litigantes que una actualización de derechos de acceso a la justicia (Albornoz 2014).

6 Los nombres de Manuel Ahumada, de Alejo Piña y de Marcelo Trincado figuran en el catastro agrícola que realizó el gobierno durante 1833 y 1834 . Los tres aparecieron en la parroquia de Guacarhue con una renta agrícola anual destinada al mercado equivalente a 18,6 pesos, respectivamente (ANHCh Fondo Contaduría Mayor 1834). Esta cifra estaba cerca del mínimo de renta, 12,4 pesos, que fijó el gobierno para ingresar a las listas de contribuyentes agrícolas (Mellafe 1988). Los datos contribuyen a deducir la pertenencia de los litigantes a una categoría de pequeños y medianos campesinos. Por ejemplo, considerando el mismo sector de Guacarhue, el catastro identificó 282 contribuyentes que reconocían una renta promedio de 49,9 pesos.

7 Esta transacción se encuentra ratificada en escritura pública. Fue elaborada por José Marful, alcalde de Guacarhue el 23 de agosto de 1831 (ARNAD, FNR 1831).

8 Este argumento cobra sentido si se considera que, desde las primeras décadas republicanas, se revalorizó lentamente la tierra en la zona central de Chile. Este factor motivó un interés social por precisar los límites de las posesiones y las ansias por definir quiénes tenían derecho al uso y goce de la tierra. La litigiosidad de estos términos, que se constataba intensa desde la centuria anterior (Albornoz 2019), se agudizó desde entonces, según devela el material disponible en los fondos judiciales civiles del ANHCh.

9 Todo indica que tras el juicio, Ahumada continuó en posesión sobre las demás tierras en disputa. La documentación notarial de Guacarhue contiene la escritura de cesión de una cuadra y media por herencia que hizo Ahumada en favor de sus cinco hijos. El documento está firmado en julio de 1834, dos meses después de finalizado el juicio (ARNAD FNR 1834). 